Assessment of Nurses' Knowledge and Skills Regarding

\title{
Oxytocin Administration
}

\author{
I Heba Mohamed AbdouWahdan Y`Dr .Om Hashim Mahmoud MahmoudSaadoon ${ }^{\Upsilon}$ Dr.HananEl-Sayed \\ Mohamed El-Sayed، \\ 1Nursing Specialist-Dammiet University, Faculty of Nursing-Mansoura University, Egypt. \\ 2Assist.Prof.of Woman's Health and Midwifery Nursing, Faculty of Nursing, Mansoura University, \\ Egypt. Corresponding author email:samarabdelhakam@gmail.com
}

\begin{abstract}
Background: Oxytocin is the drug more commonly used for mother during labour for induction and progression of labour process but misuse of oxytocin administration results in serious adverse effects to mother and baby. Aim: To assess nurses' knowledge and skills regarding oxytocin administration. Design: A cross-sectional descriptive study design was used. Setting: The study was conducted at labor and delivery Unit in Mansoura University Hospitals, New General Hospital and Old General Hospital. Sample type: A convenient sample was utilized. Sample: Sixty nurses who work in the labor room of selected hospitals. Tools: Tool I: Structured Interview Schedule: It includes general characteristics of nurse and knowledge of nurses about oxytocin administration. Tool II: Oxytocin administration observational checklist Results: All of studied group (100\%) had knowledge about time to stop the oxytocin infusion during labor; most of them had knowledge regarding the interval that oxytocin dosage increased. Also, majority of them had knowledge regarding the appropriate action to be performed if the uterine hyper stimulation occurs during oxytocin administration. While three quarters of the studied group had deficiency knowledge about the appropriate duration of oxytocin action. Regard to nurses' practice in administration of oxytocin; all of studied group (100\%) completely connects the woman with fetal monitor and record intake and output. Else, majority of them completely prepare equipment, while around half of studied group not had done wash hands post procedure and no one complete the partogram. Conclusion: More than two third of the studied group had average level of knowledge about administration of oxytocin, more than half of studied group have good practice in administration of oxytocin also, there was a positive moderate significant correlation between knowledge and practice. Recommendation: Conducting periodic in-services training program for nurses who are handling oxytocin drugs to continuous improve knowledge and skills for safe handling measures and reduce the harmful effects of this drug
\end{abstract}

Keywords: Augmentation, induction, knowledge, labor, oxytocin, skills 
Heba Mohamed AbdouWahdan et. al.

\section{Introduction}

Labor is considered a life changing event to the mothers and their families all over the world, the time of birth is very important to the survival of women and their babies, as the risk of morbidity and mortality could increase considerably if complications arise. Enhancing the quality of care around the time of birth has been describing as the most effective approach for reducing stillbirths, maternal and neonatal deaths, compared to antenatal or postnatal care intervention (WHO, 2020).

Induction of labor is the process of stimulating uterine contractions to initiate labor and achieve delivery (Paul Lyons Nathan McLaughlin, 2020). Endogenous oxytocin has an important role in the physiological progress of labour. It is a peptide hormone, produced in the hypothalamus and released into the blood circulation in a pulsative manner. During child birth, the release of oxytocin increases. It plays an important role during labour by stimulating the frequency and intensity of uterine contraction and cervical dilation. In delayed labour it used to shorten labour duration to prevent adverse labour outcomes, such as cesarean section or instrumental vaginal delivery (Selin, 2018).

Oxytocin is known to be the most widely used drug to induction of labour as it is a safe medication. Oxytocin is a labor induction medication used when there is failure of cervical dilatation and fetal descent with sudden uterine contraction that requires good monitoring avoid complications such as fetal respiratory distress due to extreme uterine contraction and uterine rupture. It also requires close monitoring to assess the labor progress and to detect signs of induction failure, if present, as soon as possible. (shiny, 2017).

Induction of labor should be carried out only by highly trained and qualified provider in facilities with access to emergency obstetric services due to increased risks of complications following these interventions (HidalgoLopezosa, Hidalgo-Maestre \& Rodríguez-Borrego, 2016). It was designated by the Center for Safe Medical Practice as a high-alert drug in 2007. High-alert medications are detected as those medications that need special considerations and precautions before and during administration which increased risk of causing significant patient harm when used improperly.

(Esmail Shady, 2020)

Different studies show how lack of protocols in the hospitals, lack of knowledge about administration of oxytocin drugs during child birth time, its monitoring and adverse side effect and inadequate provision for in-service education for the midwifery nurse lead to improper oxytocin administration. Good understanding of the oxytocin induction during labour leads to reducing complications of improper knowledge and skills of oxytocin induction. (Soman, et al., 2019)

Related to the International Confederation of Midwives (ICM) model of midwifery care, midwives are responsible for promoting and protecting women's and newborns' health and rights and for enhancing and advocating non-intervention in normal childbirth (Butler, et al, 2018).

Administration of oxytocin drug during labor need close nursing monitoring and good observation, midwifery nurse should be have a sound knowledge and skills about nursing care 
to woman who is receiving an oxytocin infusion, so it needs good nursing monitoring to assess the labor progress and to identify signs of failure of induction as soon as possible, give efficient nursing care to prevent complications such as fetal respiratory distress and hyper stimulation related to severe uterine contraction. (Mergani, 2015).

Studies on knowledge and skills during administration of oxytocin among midwifery nurses showed that two third of the studied group had poor knowledge about use of oxytocin, Also regard to practice majority of nurses had unsatisfactory practice (Esmail Shady et al, 2020) therefore, the investigator motivated to assessment of nurses knowledge and skills during administration of oxytocin in selected hospitals.

\section{Significance of the study}

Safe childbirth is the responsibility of the midwifery nurses by enhancing and ensuring the health of the mother and fetus wellbeing. During labor, a group of drugs called "oxytocics" are commonly given to women during labor. On the basis of their nursing assessment, nurses at the bedside of mother make a recommendation on oxytocin titration. Improper oxytocin has been reported as leading to maternal and neonatal mortality. Improper uses of oxytocin by insufficiently knowledgeable and qualified member. The stakes are high, with the unsafe use of such medicine having significant implications for mother and child. (Murray, Nelson \& Ashwill, 2017; Esmail Shady et al, 2020).

Studies about knowledge and practice during oxytocin administration intervention among maternity nurses showed that staff nurses had below average level of knowledge and poor level of practice during administration (Metwally et al, 2019).

Also, the investigator while working in hospitals found that nurses were generally unable to work in other wards other than the maternity unit because they were not confident about the use of different medicines and procedures in their area. So the investigator decided to conduct this research.

\section{Aim of the study}

This study aims to assess nurses' knowledge and skills regarding oxytocin administration.

\section{Research Questions}

$>$ What are nurses' knowledge and skills regarding oxytocin administration?

$>$ Is there a significant relationship in selected hospitals between knowledge and skills regarding the administration of oxytocin among nurses?

\section{Subjects and method}

Study Design: A cross-sectional descriptive study design was used.

Study Setting:

The study was conducted in three hospitals as (Labor and delivery Unit at Mansoura University Hospitals which consisted of room for examination, a big room consists of six beds, a room for pre-eclampsic patient, a room for ultrasound, and postnatal room. Nearly more than 80 cases per month visit the unit for delivery in three days per week (Sunday- Tuesday- Thursday). Labor and delivery Unit at New General Hospital which consists of 2 operation room, 8 post-natal rooms each one consisted of 3 beds, admission 2 days per week (Saturday-Wednesday), and nearly more than 30 cases per month visit the unit for 
delivery. Labor and delivery Unit at Old General Hospital which consisted of examination room, operating room, and 3 postnatal room 3 beds in each one, admission 2 days per week (MondayFriday), nearly more than 30 cases per month visit the unit for delivery.

Sample type: A convenient sample was used.

Study Sample: All nurses who work in the labor and delivery unit of predetermined setting regardless their age, experiences or qualification and their number are (60).

Sample size: Total coverage because the number of the nurses is limited.

Tools for data collection: Data was collected using two tools

Tool I:Structured Interview Schedule: It was developed by the investigator after extensive review of the relevant national and international literature. It consisted of two parts:

Part(1):General characteristics of nurses such as age, level of education, years of experience, any training course, residence data ....etc.

Part (11): Knowledge of nurses about oxytocin administration:

It addressed nurses' knowledge regarding the definition of oxytocin, uses, action, protocol oxytocin infusion during labor, knowledge of nurses about subsequent doses of oxytocin and how many units in the initial dose of oxytocin infusion ...etc. Every correct answer was given a score of "one" mark, wrong answer "zero" score. The total knowledge score was below average if $<$ $50 \%$, Average if $50<75 \%$ and above average if $\geq 75 \%$.

Tool II: Oxytocin Administration Observational Checklist: It was developed by Health Workforce Development, 2006. It was obtained from maternity and gynecologic nursing clinical checklist; it adopted from Egypt health workforce project (2006). It was used to assess nurse's clinical skills level related to oxytocin administration. It consisted of three domains and 19 items. Getting Ready (8 items), management of oxytocin infusion steps (6 items) and post procedure tasks ( 5 items). Each item was scored (2) if completely done, scored (1) if incompletely done, and scored (0) if not done. The total score was ranged from 19 to 58; higher score indicates higher achievement.

Validity of the study tools:

Content validity of data collection tools was determined through an extensive review of literatures about oxytocin administration. Also, tools were tested and juried for their validity by five specialists in the field of woman's health and midwifery nursing and the recommended modifications were done. As grouping questions about knowledge of nurses regard oxytocin administration in domains to make the evaluation more specific and clear.

Reliability of the tools:

Internal consistency and a reliability coefficient (Cronbach's alpha) of the components of the questionnaire tested by SPSS software. Cronbach's alpha for knowledge questionnaire is (0.706) and it was $(0.715)$ for tool II observational checklist. This means good reliability of the tools.

\section{Pilot study:}

The pilot study was conducted with $10 \%$ (6 nurses) of the studied group to evaluate the clarity and applicability of the tools, as well as to estimate the time needed for answer. The results of the pilot study didn't included in the sample size and according to analysis of pilot results, modifications of the tools were done as paraphrasing of some sentences. 
Ethical considerations

An ethical approval was taken

from the Ethics Committee of the

Faculty of Nursing, Mansoura University, an official permission was obtained from the director of predetermined setting to obtain the official permission for conduct the study after explaining the aim of the study, prior to the study, informed consent was obtained from each nurse after providing an explanation of the nature, purpose of the study, the benefits, and risks, the investigator was emphasize to participants that participation is voluntary and any participant was permitted to withdraw from the study at any time without any reason, confidentiality was assured throughout the whole study, the results was used for the purpose of publication and education only.

\section{Fieldwork:}

- The actual fieldwork of the study was conducted for 6 month's period from beginning of September 2019 to end of February 2020.

- Official permission was obtained from the Mansoura University Hospitals director , New General Hospital and Old General Hospital; head of woman's health and midwifery nursing department and from the faculty of nursing ethical committee to perform the study .

- The investigator attended to each labor and delivery unit from 9 A.m. to 5 P.m according to hot days' in each hospital as Mansoura University Hospitals attended to the unit in three days per week (SundayTuesday- Thursday). Labor and delivery Unit at New General Hospital two days per week
(Saturday-Wednesday), Old General Hospital admission two days per week (Monday-Friday).

- The investigator introduced her to the nursing staff and obtains their consent to participate in the study after explanation of the aim Obviously,.

- The nurses informed of the voluntary nature of the study. After they provide informed consent.

- The investigator interviewed each nurse individually in private setting to collect general characteristics and fill knowledge about oxytocin by asking questions and recording the answers each nurse take from (1520) minute to answer the questions. After that, the researcher assessed nursing staff skills about oxytocin administration using an observational checklist.

- The investigator attended to each labor and delivery unit until the sample number completed.

\section{Data Analysis}

Collected data were coded, computed and statistically analyzed using SPSS (statistical package of social sciences), version 16. Data were presented as frequency and percentages (qualitative variables) and mean $\pm \mathrm{SD}$ (quantitative continuous variables). Chi square $(\chi 2)$ was used for comparison of categorical variables, and was replaced by Fisher exact test (FET) or Mont Carlo Exact test if the expected value of any cell was less than 5. Pearson correlation test (r) was used to find correlation between two continuous quantitative variables. The difference was considered significant at $\mathrm{P} \leq 0.05$ 
Heba Mohamed AbdouWahdan et. al.

Table (1): Distribution of the studied group according to their socio demographic characteristics

\begin{tabular}{|l|l|c|c|}
\hline \multicolumn{1}{|c|}{ Characters } & \multicolumn{1}{|c|}{ Items } & no (60) & $\%$ \\
\hline Age (years) & $18-23$ 13 & 21.7 \\
& $24-29$ & 15 & 25.0 \\
& $30-35$ & 17 & 28.3 \\
& $>35$ & 15 & 25.0 \\
\cline { 2 - 4 } & \multicolumn{2}{|c|}{ Mean \pm SD $=31.58 \pm 8.73$ years } \\
\hline \multirow{2}{*}{ Education } & Nursing Deplume & 22 & 36.7 \\
& Technical Institute & 36 & 60.0 \\
& Nursing Bachelor & 2 & 3.3 \\
\hline Job & Staff nurses & 57 & 95.0 \\
& Head nurse & 3 & 5.0 \\
\hline Residence & Rural & 44 & 73.3 \\
& Urban & 16 & 26.7 \\
\hline Training on oxytocin & Yes & 0 & 0.0 \\
& No & 60 & 100.0 \\
\hline
\end{tabular}

Table(1) shows distribution of the studied group according to their socio demographic characteristics. It was found that mean age of nurses was $(31.58 \pm 8.73)$ years. $60 \%$ of them had technical institute and most of them (95\%) working as staff nurse. Around three quarters of nurses $(73.3 \%)$ from rural areas. No one of them obtained training on oxytocin administration.

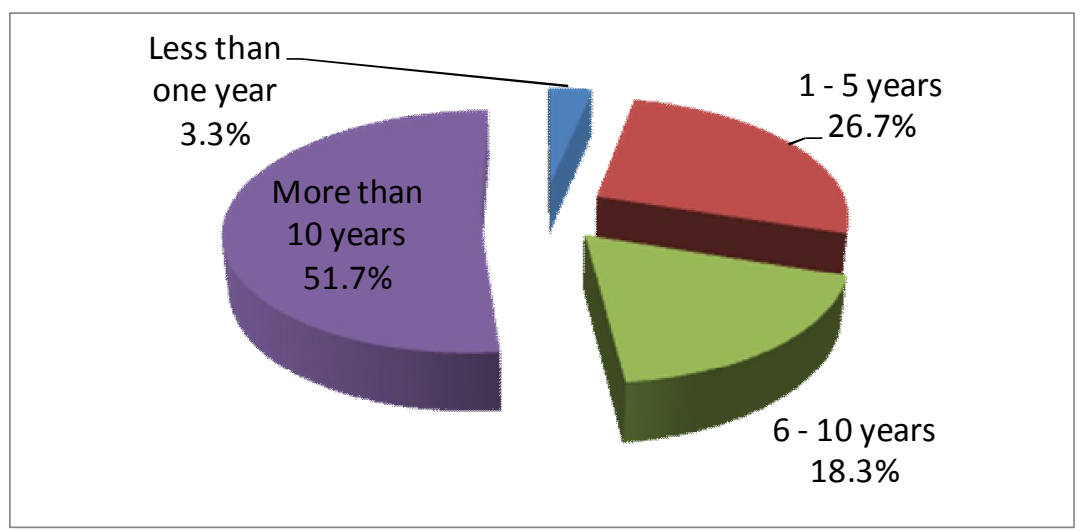

Figure (1): Distribution of the studied nurses according to their experience years as a nurse. 


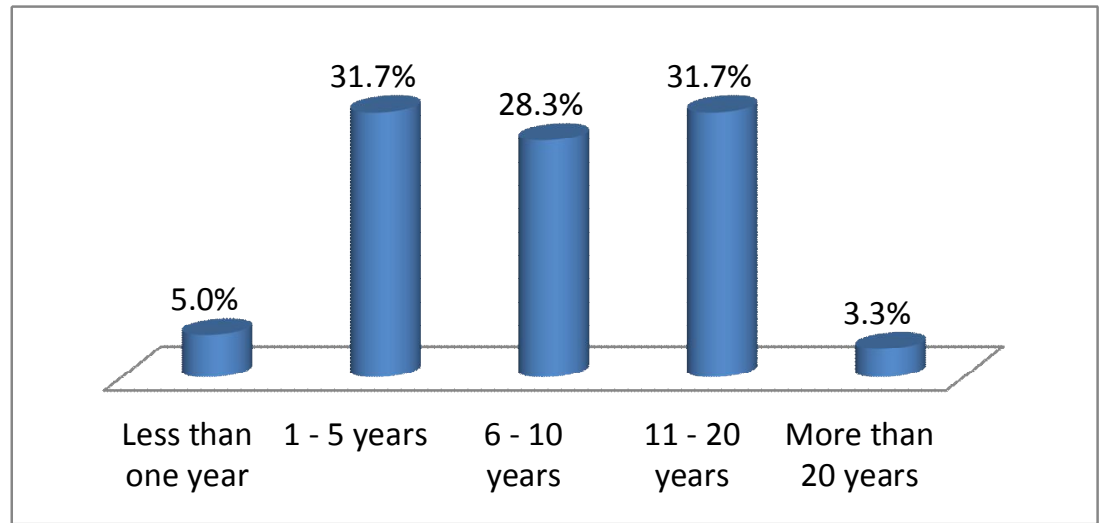

Figure (2): Distribution of the studied nurses according to their experience years in maternity care.

Table (2): Knowledge level of the studied group regard oxytocin administration.

\begin{tabular}{|c|c|c|c|c|c|c|}
\hline \multirow[t]{2}{*}{ Domains } & \multicolumn{2}{|c|}{$\begin{array}{c}\text { Below average } \\
\text { Knowledge } \\
(<\mathbf{5 0 . 0} \%)\end{array}$} & \multicolumn{2}{|c|}{$\begin{array}{c}\text { Average } \\
\text { Knowledge } \\
(\mathbf{5 0 . 0}-<\mathbf{7 5 . 0 \% )}\end{array}$} & \multicolumn{2}{|c|}{$\begin{array}{c}\text { Above average } \\
\text { Knowledge } \\
(\geq 75.0 \%)\end{array}$} \\
\hline & No & $\%$ & No & $\%$ & No & $\%$ \\
\hline $\begin{array}{l}\text { Knowledge regarding definitions, uses and } \\
\text { routes of oxytocin }\end{array}$ & 16 & 26.7 & 41 & 68.3 & 3 & 5.0 \\
\hline $\begin{array}{l}\text { Knowledge regarding dose, action and duration } \\
\text { of oxytocin administration }\end{array}$ & 31 & 51.7 & 24 & 40.0 & 5 & 8.3 \\
\hline $\begin{array}{l}\text { Knowledge regarding observation and monitor } \\
\text { mother \& fetus }\end{array}$ & 6 & 10.0 & 41 & 68.3 & 13 & 21.7 \\
\hline $\begin{array}{l}\text { Knowledge regarding oxytocin side effects, } \\
\text { complications and contraindications }\end{array}$ & 15 & 25.0 & 45 & 75.0 & 0 & 0.0 \\
\hline $\begin{array}{l}\text { Knowledge regarding how to manage side } \\
\text { effects, complications }\end{array}$ & 11 & 18.3 & 40 & 66.7 & 9 & 15.0 \\
\hline Total Knowledge & 20 & 33.3 & 40 & 66.7 & 0 & 0.0 \\
\hline
\end{tabular}

Table (2) shows the knowledge level of the studied group for different domains of the tool assessing their knowledge about oxytocin administration. Average knowledge level was $(68.3 \%)$ in first domain, $(40.0 \%)$ in second domain, $(68.3 \%)$ in third domain, $(75.0 \%)$ in fourth domain and $(66.7 \%)$ in the fifth domain. While knowledge level was below average in these domains being $(26.7 \%)$ in first domain, $(51.7 \%)$ in second domain, $(10.0 \%)$ in third domain, $(25.0 \%)$ in fourth domain and $(18.3 \%)$ in the fifth domain. Moreover, above average knowledge was $(5.0 \%)$ in first domain, $(8.3 \%)$ in second domain, $(21.7 \%)$ in third domain, $(0.0 \%)$ in fourth domain and $(15.0 \%)$ in the fifth domain. 
Heba Mohamed AbdouWahdan et. al.

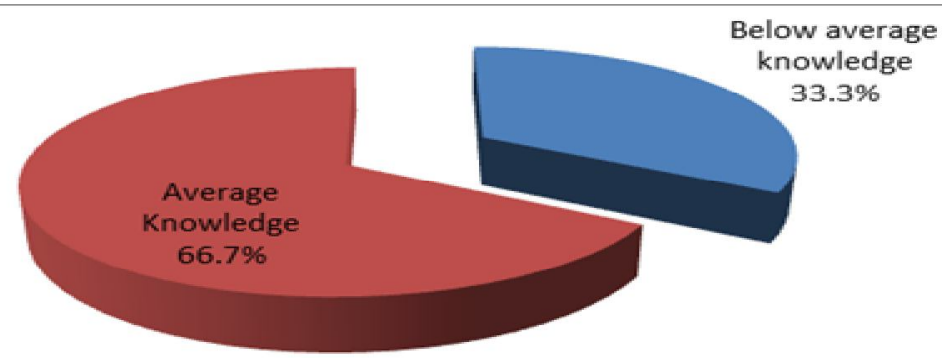

Figure (3): Level of knowledge of the studied group about oxytocin administration.

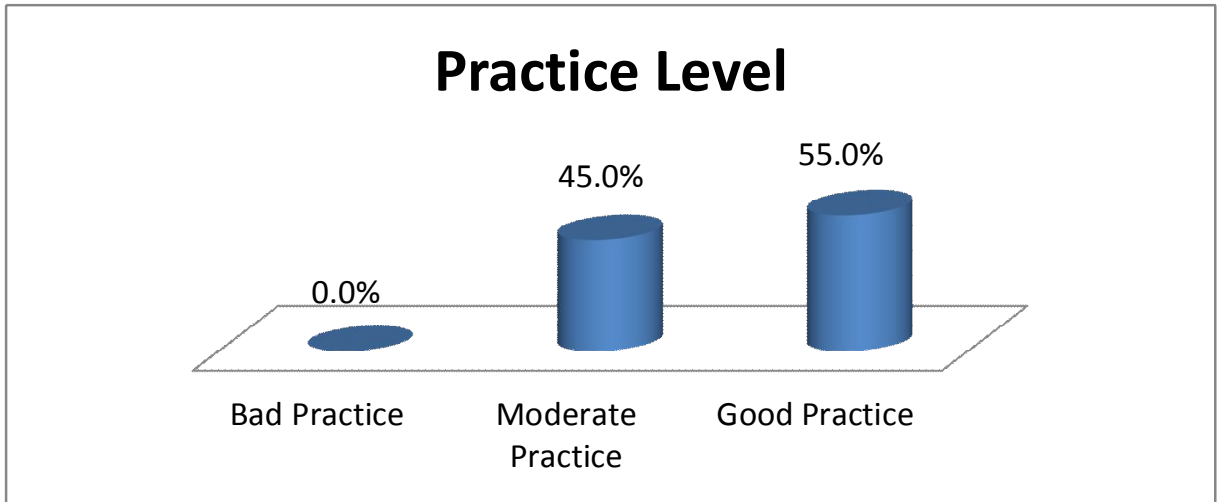

Figure (4): Level of practice of the studied group regard management of oxytocin administration.

This study aimed to assess nurses' knowledge and skills regarding oxytocin administration. The study research questions were supported by the study findings, such findings evidenced that the total knowledge level was average in more than two third of the studied group, Also more than half of studied group had good practice, else, there was a positive, moderate significant correlation existing between knowledge and practice.

Regarding to level of nurses knowledge about oxytocin administration, the study findings showed that the total knowledge level was average in more than two third of the studied group, below average in one third and no one had above average level in total knowledge, these findings were supported by the study conducted by Soman et al., (2019) they reported that more than half of studied nurses had moderate level of knowledge regarding to oxytocin administration. Also, this finding was in the same line with Mergani (2015) who reported that more than two third of the staff nurses had adequate knowledge.

While the present study findings were disagree with the results by Lohani, (2020) who reported that majority of the studied nurses had good knowledge. These results were in the same direction with the study by Esmail Shady, Gamal, Ebrahem, (2020) about assessment of nurses compliance with 
oxytocin administration protocol during labor at dammieta city and showed that two third had low level of knowledge about oxytocin administration, they denoted that majority of them had below average level of knowledge. Similar finding was reporting in the study by Shrestha \& Semmalar (2017) about the effectiveness of self-instructional module on knowledge regarding maternal and neonatal outcome of induction of labour among staff nurses in selected hospital, Bangalore, India and reported that two third of them had inadequate level of knowledge.

Moreover, the current results in contrast to the results were observed by Shiny (2017) who indicated that two third of the participants had below average level of knowledge. Else, Ahla, Fatihah \& Norziyana (2015) they performed a study to assess the knowledge of nurse's midwives regarding nursing care of a women receiving oxytocin drug during labour in El Ribat, they stated that less than one third of staff nurses had sufficient pretest knowledge.

Furthermore, the present study results were in disagreement with Roma et al., (2014) they indicated that about two third of the studied nurses had poor level of knowledge related to oxytocin administration protocol, Also, the present study findings were not in agreement with Haleena \& Prathap (2013) they evaluate the knowledge and practice of oxytocin induction and quality of delivery care among staff nurses working in maternity ward they reported that two third of nurses had insufficient knowledge.

As regard level of practice about administration of oxytocin among the studied group. The current study findings showed that good practice was recorded in more than half of the studied group, moderate practice in less than half and no one has bad practice level. The present finding was not surprising as most of them had experience years in maternity care range from 1 up to 20 years and two third of them had technical institute. The present study findings were in disagreement with Lohani (2020) who reported that more than two third of the studied nurses had moderate level of practice, one third of them had poor level of practice, but no one had good level of practice regarding oxytocin administration intervention.

Also, the current study findings were not in same line with a study done by Esmail shady et al., (2020) they revealed that most of studied nurses had unsatisfactory practice after women induction with oxytocin, while one third only of them had satisfactory practice before women induction with oxytocin, regarding to total score of practice majority of them had unsatisfactory practice level. Also the current study results were in contract with Metwally et al., (2019) they indicated that staff nurses had poor level of practice during oxytocin administration. Also, Shiny (2017) shows that two third of the staff nurses had poor practice, one third of the staff nurses had moderate practice level and less than one quarter of the staff nurses had good practice during administration of oxytocin.

Regarding the relationship between knowledge and practice among the studied group it was reported that there was a positive, moderate significant correlation existing between knowledge and practice. The above results were approved by the study presented by Lohani (2020) who show a positive correlation between knowledge and practice. Furthermore, a study conducted 
by Shiny (2017) showed that there was a positive correlation between knowledge and practice,

\section{CONCLUSION}

More than two third of the studied group had average level of knowledge about administration of oxytocin. Also, more than half of studied group have good practice in administration of oxytocin. There was a positive, moderate significant correlation between knowledge and practice.

\section{RECOMMENDATIONS}

- Conducting periodic in-services training program for maternity nurses who are handling oxytocin drug to continuous improve knowledge and skills for safe handling measures and reduce the harmful effects of this drug.

- Clinical demonstration should be performed regarding the administration of oxytocin to enhance the practical skills level of maternity nurses.

- Further studies are recommended to investigate the effect of educational sessions about oxytocin on midwives nurses' knowledge and practice.

\section{Acknowledgement}

The investigator would like to thank all participants for their cooperation during the study.

\section{CONFLICTS OF INTEREST.}

The investigators declare that there is no conflict of interest statement.

\section{References}

1- Ahla, Fatihah \&Norziyana (2015): Assessment of nurse's midwives knowledge regarding nursing care of a woman who receiving oxytocin drug during labor, Ribat University, Faculty Graduate Studies \& Scientific Research
2- Butler, M. M., Fullerton, J. T., \& Aman, C. (2018). Competence for basic midwifery practice: Updating the ICM essential competencies. Midwifery, 66, 168175

3- Esmail Shady, R. R., Ibrahem, S. S., Gamal, A. M., \& Farahat, F. Z. (2020). Assessment of nurses' compliance with oxytocin administration protocol during labour at Dammita city. Port Said Scientific Journal of Nursing, 7(3), 42-65.

4- Haleena\&Prathap, V (2013): Retrospective study of assess the knowledge and practice regarding oxytocin induction and quality of delivery care

5- Hidalgo-Lopezosa, P., HidalgoMaestre, M., \& RodríguezBorrego, M. A. (2016). Estimulação do parto com oxitocina: efeitos nos resultados obstétricos e neonatais. Revista Latino-Americana de Enfermagem,.; 24: e2744.

6- KP, D., Soman, B., Wilson, K., Nandagaon, J. G., Joseph, P., \& Aman, J. (2019). "Are the Maternity Nurses Knowledgeable on Oxytocin Induction During Labour? A Protocol Development for Hospitals in South India". Indian Journal of Public Health Research \& Development, 10(8)

7- Lohani, B. (2020). Nurses knowledge and practice regarding oxytocin administration during first stage labor in maternity ward of teaching hospital. Journal of Patan Academy of Health Sciences, 7(2), 103-11

8- Lyons, P., \& McLaughlin, N. (2020). Induction and Augmentation. In Obstetrics in 
Family Medicine (pp. 169-171). Humana, Cham.

9- Mergani, R. (2015). Assessment of nurse $\mathrm{s}$ midwives knowledge regarding nursing care of a woman who receiving oxytocin drug during labor Ribat University, Faculty Graduate Studies \& Scientific Research.

10- Mohamed,A ., Desoky, M., Metwally,H.(2019). Effect of educational program on improving nursing knowledge and practice regarding administration of oxytocin during labor. IOSR Journal of nursing and health science. 8(5), 44-53.

11- McKinney, E. S., James, S. R., Murray, S. S., Nelson, K., \& Ashwill, J. (2017). Maternal-child nursing-e-book. Elsevier Health Sciences

12- Roma, N., Al-Battawi, JN., \& Zaki, N. (2014). Nurses' Compliance with oxytocic administration guidelines during labor . Alexandria Scientific nursing Journal, 16(2), 55-72
13- Selin, L. (2018). Delayed labourrisk factors, use of oxytocin and outcomes niversity of Gothenburg. Sahlgrenska Academy Fredagen den 21 september $2018 \mathrm{kl} 09.00$, Hörsal, Medicinargatan.

14- Shiny, S. T. (2017). Assessment of the Knowledge and Practice on Use of Oxytocin among Nurses Working in Selected Hospitals in Chennai (Doctoral dissertation, MA Chidambaram College of Nursing, Chennai)

15- Shrestha, R. (2017). The effectiveness of self-instructional module on knowledge regarding maternal and neonatal outcome of induction of labour among staff nurses in selected hospital, Bangalore, India. Journal of advanced academic research, 4(2), 79-91.

16- World Health Organization. (2020). WHO labour care guide: user's manual. World Health Organization.

https://apps.who.int/iris/handle/10 665/337693. License: CC BY-NCSA 3.0 